\title{
Evaluation of the nasal microbiota in slaughter-age pigs and the impact on nasal methicillin-resistant Staphylococcus aureus (MRSA) carriage
}

\author{
J Scott Weese ${ }^{1 *}$, Mackenzie Slifierz', Mohammad Jalali and Robert Friendship ${ }^{2}$
}

\begin{abstract}
Background: The nasal microbiota of pigs has been poorly assessed but could play a role in carriage of important microorganisms such as methicillin-resistant Staphylococcus aureus (MRSA). The objectives of this study were to describe the nasal microbiota in slaughter age pigs, to evaluate the impact of farm management on the nasal microbiota and to provide a preliminary assessment of the influence of the microbiota on MRSA carriage.

Results: Nasal swabs were collected from five MRSA positive and eight MRSA negative pigs on one farm that used a liquid feeding system and routine tylosin treatment, and seven MRSA negative pigs from an antibiotic-free farm that used conventional feeding. A total of 946310 sequences passed all quality control filters. The number of sequences per sample ranged from 4307 to 165656 (mean 56092, SD 40007). CatchAll analysis of richness predicted a mean of 1749 OTUs (range 213-3736, SD 996). Overall, 6291 OTUs were identified, yet 5125 (81\%) were identified less than 10 times and the 12 most abundant OTUs accounted for $80.7 \%$ of sequences. Proteobacteria predominated in all but two samples. Liquid-fed/tylosin-exposed pigs had significantly lower relative abundances of Verrucomicrobia $(P=0.004)$, Fibrobacteres $(P=<0.0001)$ and sequences unclassified at the phylum level $(P=0.028)$. When comparing only liquid-fed pigs, MRSA carriers had significantly more Bacteroidetes $(P=0.037)$ than MRSA negative pigs. 124 genera were identified, with Moraxella accounting for $35.4 \%$ of sequences. In the Jaccard index tree, five of eight MRSA positive pigs clustered closely together, as did six of the seven conventionally-fed pigs. A significant difference was identified between conventional and liquid-fed pigs using parsimony test with the Jaccard $(P<0.001)$ but not the Yue\&Clayton $(P=0.26)$ index. There were no significant differences between MRSA positive and negative pigs ( $P=0.133$ and 0.175$)$. OTUs belonging to Firmicutes were the main indicators of MRSA negative pigs, including Lactobacillus and another Lactobacillaceae and Staphylococcus.
\end{abstract}

Conclusions: Farm management can influence the nasal microbiota in pigs, but no impact of the microbiota on MRSA carriage was identified. Studies that further define the impact of management on the microbiota, and the impact of the microbiota on pathogen carriage are indicated.

Keywords: Microbiota, Antimicrobial resistance, Staphylococci

\section{Background}

The body contains numerous different ecological niches that support abundant and diverse populations of microorganisms. The microbial composition of a body site (the microbiota) and the sum of its genetic materials (the microbiome) are of increasing interest since it is clear that they can play critical roles in health and disease. Recent

\footnotetext{
* Correspondence: jsweese@uoguelph.ca

'Department of Pathobiology and Centre for Public Health and Zoonoses, Ontario Veterinary College, University of Guelph, Guelph, Ontario N1G2W1, Canada Full list of author information is available at the end of the article
}

advances in next generation sequencing and bioinformatics have resulted in the ability to characterize complex microbial populations at a level that was impossible only a few years earlier. These studies are providing remarkable insight in to the body's microbial populations and their interaction with the host.

Most microbiota studies have focused on the intestinal tract or feces, but various other body sites harbour important microbial populations, including the nasal passages. There has been limited investigation of the nasal microbiota in pigs, with culture-based studies focusing on 
selected pathogens [1]. There has been some study of the microbiota of tonsillar tissue. A study of 18-20 week old pigs, bacteria from the Proteobacteria phylum predominated, with Firmicutes and Fusobacteria (phyla) being relatively common and 14 other phyla being present but rare [2]. It is likely that the tonsillar and nasal microbiotas are similar, but this has not been investigated.

While there has been limited study, the nasal microbiota may be of relevance for many reasons, including understanding of the ecology of important pig and zoonotic pathogens. One area of potential importance is methicillin-resistant Staphylococcus aureus (MRSA) colonization. Since 2005 [3], MRSA has become an important concern in pigs. While rarely a cause of disease in pigs, MRSA is a human health concern. High rates of MRSA carriage have been reported in pigs internationally [4-6] and pig contact is a leading source of MRSA exposure in people in some countries [7]. While MRSA carriage rates can be very high on farms, not all pigs that are exposed to MRSA become colonized. Reasons for this are unclear but one possibility is a protective effect of the endogenous nasal microbiota. If true, this could represent a potential MRSA control intervention by modification of the nasal microbiota to reduce the likelihood of MRSA carriage.

The objectives of this study were to describe the nasal microbiota in slaughter age pigs, to evaluate the impact of diet on the nasal microbiota and to provide a preliminary assessment of the potential influence of the microbiota on MRSA carriage.

\section{Results}

Initial screening of 100 pigs from two farms resulted only in identification of farms where all or no pigs were carrying MRSA. Both MRSA positive $(n=5)$ and negative $(n=8)$ pigs were subsequently identified on a farm that used a liquid feeding system that consisted of corn, wheat shorts, soybean and whole whey. Pigs also receive tylosin until the time of slaughter. As a comparison, seven MRSA-negative pigs were enrolled from one farm that used conventional feeding practices. Pigs from this farm were not exposed to antimicrobials at any time in their lives.

A total of 946310 V4 16S RNA gene sequences passed all quality control filters. The number of sequences per sample ranged from 4307 to 165656 (mean 56092, SD 40007). CatchAll analysis of richness predicted a mean of 1749 OTUs (range 213-3736, SD 996). Significantly higher OTU richness was present in the liquid-fed group (mean 2150, SD 879) versus the conventionally-fed group (mean 1005 , SD 777) $(P=0.0098)$. There was no difference between MRSA positive and MRSA negative pigs $(P=0.78)$. However, when Catchall analysis was repeated using a subsample of 4307 sequences per sample, no significant differences based on feed group or MRSA status $(P=0.38$ and 0.69 , respectively).
Overall, 6291 OTUs were identified, yet 5125 (81\%) were identified less than 10 times and the 12 most abundant OTUs accounted for $80.7 \%$ of sequences. Nine different phyla were identified; however, only three (Proteobacteria, Firmicutes, Spirochaetes) accounted for $>1 \%$ of sequences. Sequences that were unclassified at the phylum level accounted for $0.1 \%$ of sequences. The Proteobacteria phylum predominated in all but two samples, in which Firmicutes accounted for the majority of sequences. The relative abundances of predominant phyla are presented in Figure 1. Liquid-fed, tylosin-treated pigs had significantly lower relative abundances of Verrucomicrobia $(P=0.004)$, Fibrobacteres $(P=<0.0001)$ and sequences unclassified at the phylum level $(P=0.028)$ compared to conventionally fed, antibioticfree pigs. When comparing only liquid-fed pigs, MRSA carriers had significantly more Bacteroidetes $(P=0.037)$ than MRSA negative pigs.

Gammaproteobacteria was the most abundant class, accounting for $78 \%$ of sequences. Twenty-one other classes were identified, with Bacilli (6.4\%), Clostridia (6.2\%), Betaproteobacteria (5.1\%) and Spirochaetes (1.1\%) accounting for at least $1 \%$ of sequences each. At the class level, liquidfed pigs had significantly fewer Fibrobacteria $(P=0.0078)$. There were no differences between MRSA positive and MRSA negative pigs.

One hundred twenty four different genera were identified, but Moraxella dominated, accounting for 35.4\% of sequences (Table 1). The next three most abundant genera were also Proteobacteria of the families Moraxellaceae, Psychrobacter (21.2\%), Pseudomonas (14.9\%) and Acinetobacter (4.8\%). Mycoplasma was found in 18 $(90 \%)$ of samples but at an overall relative abundance of only $0.1 \%$. Genera that were present at significantly different relatively abundances between liquid- and conventionally-fed pigs are presented in Table 2 An unclassified Burkholderiales $(\mathrm{P}=0.02)$ and Comamonas $(\mathrm{P}=0.047)$ were more abundant in MRSA positive pigs compared to MRSA negative pigs.

While Staphylococcus sequences were identified in every sample, they were uncommon, ranging from $<0.01-8.6 \%$ (mean $0.54 \%$, SD 2.2\%). The relative abundance of Staphylococcus was higher in the conventionally fed pigs compared to liquid-fed, tylosin-exposed MRSA shedders $(P=0.02)$ but there was no difference overall between liquid- and conventionally fed pigs $(P=0.96)$ or MRSA positive vs MRSA negative liquid-fed pigs $(P=0.52)$.

Fifteen different genera belonging to the Enterobacteriaceae family were identified (Escherichia, Pantoea, Serratia, Yersinia, Enterobacter, Buttiauxella, Citrobacter, Hafnia, Morganella, Obesumbacterium, Proteus, Providencia, Rahnella, Salmonella and an unclassified genus), but only at abundances ranging up to $0.25 \%$. Salmonella was identified in $4 / 7$ (57\%) pigs from the conventionally fed farm but none from the liquid fed farm. 


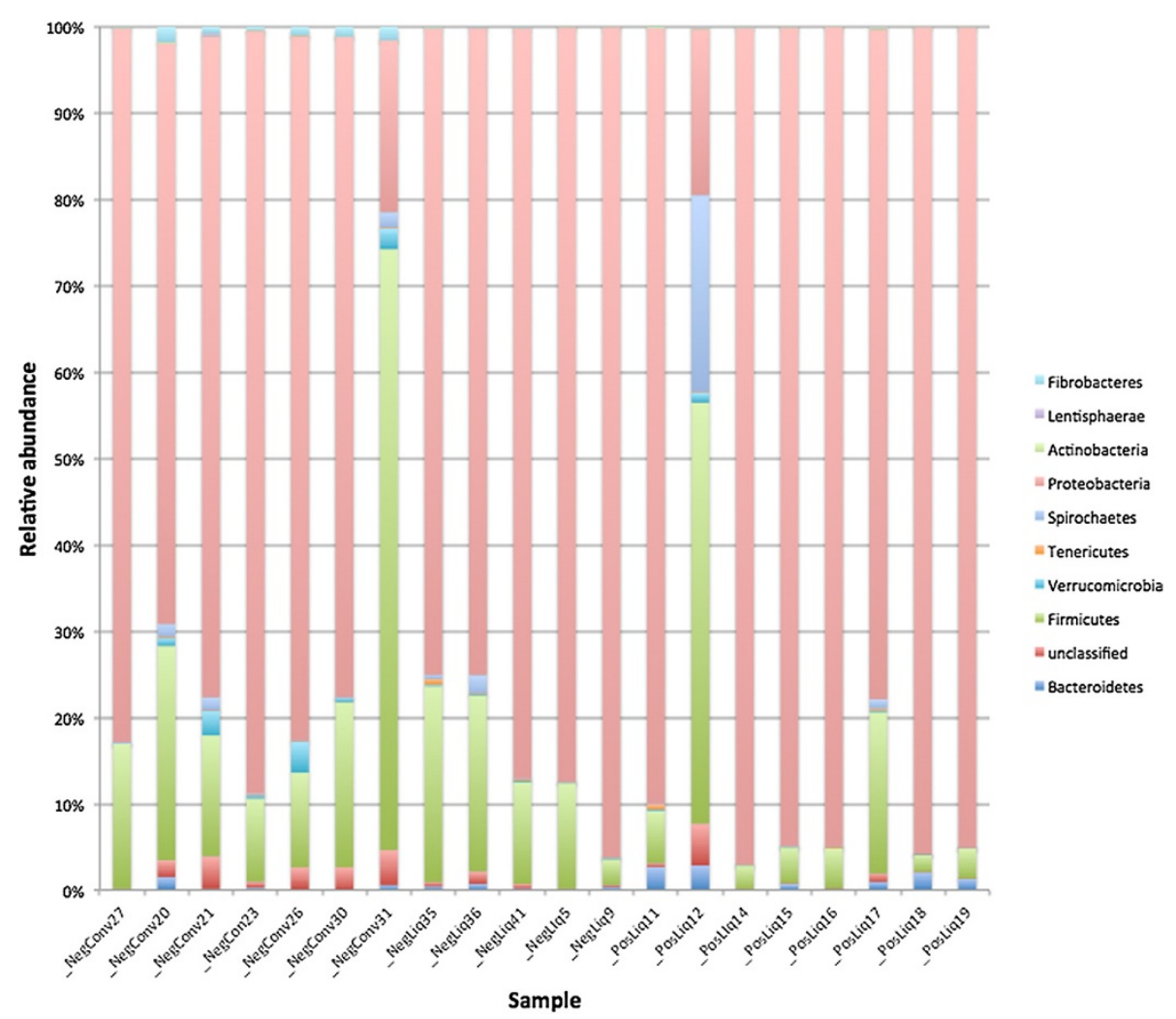

Figure 1 Relative abundances of bacterial phyla from nasal swabs from twenty pigs.

Further analysis was performed on random subsampling of 4307 sequences per sample. Rarefaction curves are presented in Figure 2. Overall, excellent sample coverage was obtained with this subsampled population, as demonstrated by a mean Good's coverage value of 0.974 (SD 0.02, range 0.92-0.99). There were no differences in coverage between groups (all $P>0.80$ ). Inverse Simpson index ranged from 1.49-40.9 (mean 5.8, SD 8.4), with no differences between groups (all $P>0.41$ ).

Table 1 Predominant genera isolated from the nasal passages of twenty healthy pigs

\begin{tabular}{lc}
\hline Genus & Relative abundance \\
\hline Moraxella & $35.4 \%$ \\
Psychrobacter & $21.1 \%$ \\
Pseudomonas & $14.9 \%$ \\
Acinetobacter & $4.8 \%$ \\
Janthinobacterium & $3.8 \%$ \\
Clostridium sensu stricto & $2.4 \%$ \\
Lactobacillus & $2.0 \%$ \\
Aerococcus & $1.8 \%$ \\
Treponema & $1.0 \%$ \\
Unclassified Ruminococcaceae & $0.9 \%$ \\
Kingella & $0.9 \%$ \\
\hline
\end{tabular}

Community structure differences can be visualized by PCA in Figure 3, as well as by dendrograms of community membership (Jaccard index) and community structure (Yue\&Clayton index) (Figures 4 and 5). In the Jaccard index tree, five of eight MRSA positive pigs clustered closely together, as did six of the seven conventionally-fed pigs. However, this was not as evident with the Yue\&Clayton index, which also assesses relative abundance.

A significant difference was identified between conventional and liquid-fed pigs using parsimony test with the Jaccard tree $(\mathrm{P}<0.001)$ but not the Yue \& Clayton tree $(P=0.26)$. There were no significant differences between MRSA positive and negative pigs $(P=0.133$ and $0.175)$. No significant differences were identified by unweighted unifrac between farms or MRSA positive and negative pigs (all $P>0.44$ ).

When comparing liquid-fed to conventionally-fed pigs, 20 significant $(\mathrm{P}<0.05)$ indicator OTUs were identified (Table 3). Eleven significant indicator OTUs were identified when comparing MRSA positive and MRSA negative pigs (Table 4).

No OTUs were present in all samples at an abundance of $1 \%$ of greater. One OTU, belonging to Moraxella, was found at this minimum relative abundance in $17 / 20$ (85\%) of sample. This OTU accounted for $20 \%$ of sequences overall. Only 3 OTUs, two Moraxella and 
Table 2 Genera that were significantly different $(P<0.05)$ in relative abundance between pigs being fed a liquid diet $(n=13)$ and those fed a conventional diet $(n=7)$

\begin{tabular}{llll}
\hline Genus & Conventional feed & Liquid feed & P value \\
\hline Unclassified Porphyromonodaceae & 0.023 & 0.007 & 0.04 \\
Macrococcus & 0.000 & 0.001 & 0.039 \\
5 genus incertae sedis & 0.014 & 0.0017 & 0.049 \\
Pseudomonas & 0.012 & 0.245 & 0.008 \\
Enhydrobacter & 0.000 & 0.0002 & 0.047 \\
Unclassified Enterobacteriaceae & 0.0047 & 0.0005 & 0.012 \\
Comomonas & 0.000 & 0.001 & 0.048 \\
Phenylobacterium & 0.00089 & 0.00028 & 0.049 \\
Corynebacterium & 0.000 & 0.00025 & 0.023 \\
Fibrobacter & 0.0093 & 0.00027 & 0.008 \\
\hline
\end{tabular}

a Psychrobacter, were identified at a relative abundance of $>1 \%$ for $50 \%$ of samples.

\section{Discussion}

This study has identified remarkable microbial richness and diversity in the porcine nasal cavity. The estimated richness (1749 species) surpasses any previous porcine study but is consistent with a human study that reported an estimate of 2264 species in the anterior nares [8]. A study of the porcine tonsil reported a smaller number of OTUs (57-730/pig) [2], but that was based on analysis of a smaller number of sequences per sample compared to the current study.
Over 6000 different OTUs were identified, yet most were rare, with 12 OTUs accounting for greater than $80 \%$ of total sequences. This shows that while the population species richness may be high, evenness is low and a few species dominate. As is common in many microbial populations, a limited number of phyla accounted for $>1 \%$ of sequences. Of the three main phyla in this population, Proteobacteria were dominant, similar to studies of the human nasal microbiota and the pig soft palate tonsil $[2,8]$. Proteobacteria includes a range of Gram negative organisms, including Moraxella (the dominant genus here), Actinobacillus and Pasteurella. While the predominance of Proteobacteria is consistent with a study of the pig

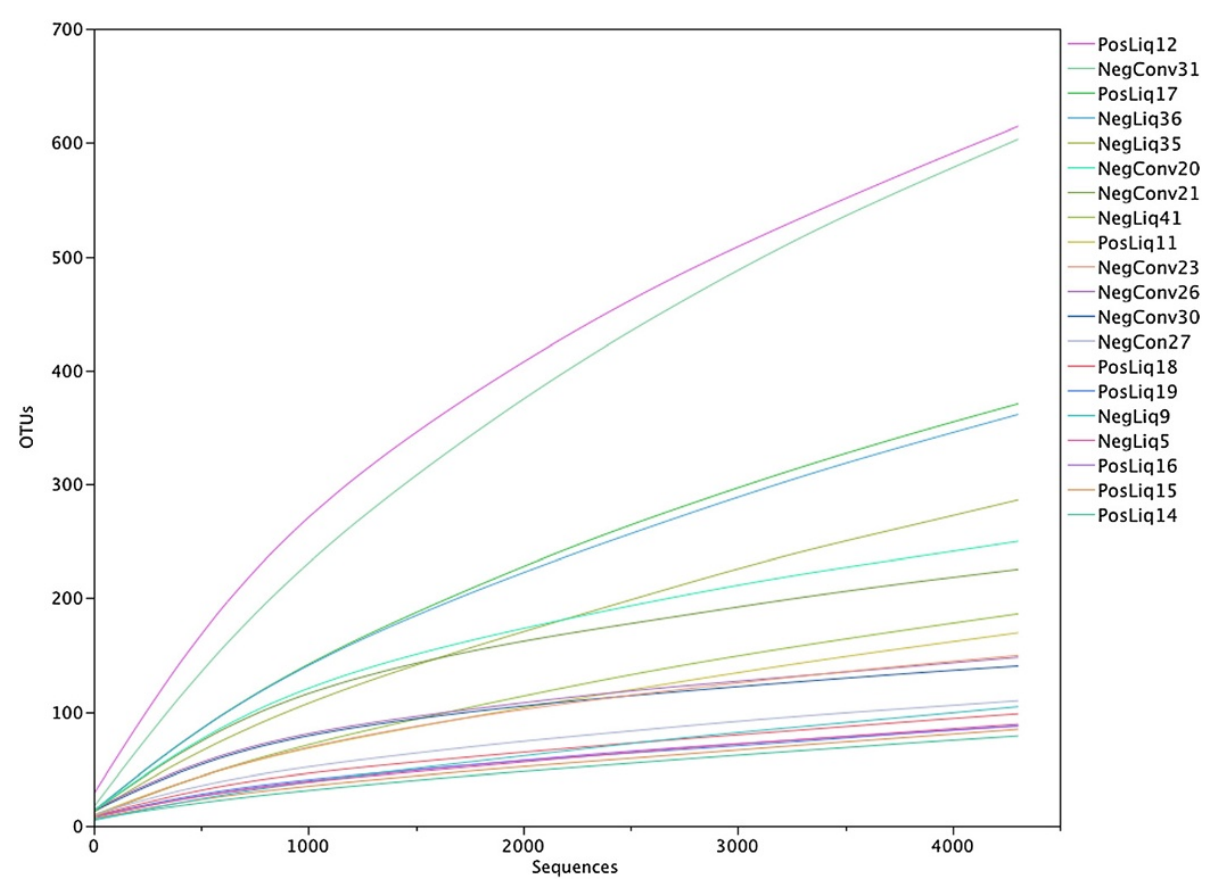

Figure 2 Rarefaction curves from a subsampled population $(n=4307)$ of V4 16S rRNA gene sequences from porcine nasal swabs. (Legend: Conv = conventionally fed pigs, NegLiq = liquid fed pigs not carrying MRSA, PosLiq = liquid fed pigs carrying MRSA). 


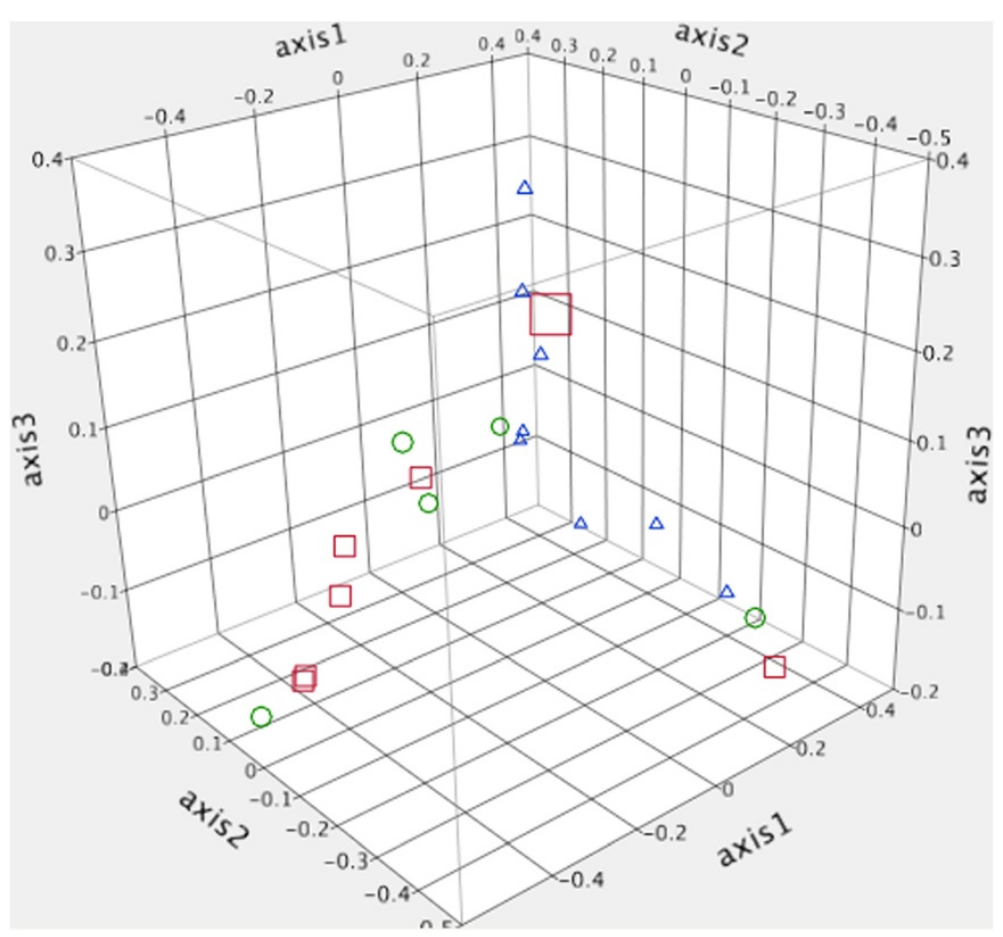

Figure 3 Three dimensional principle component analysis of the nasal microbiota of twenty healthy pigs. (Red square $=$ conventionally fed pigs, Blue triangle = MRSA negative liquid fed pigs, Green circle = MRSA positive liquid fed pigs).

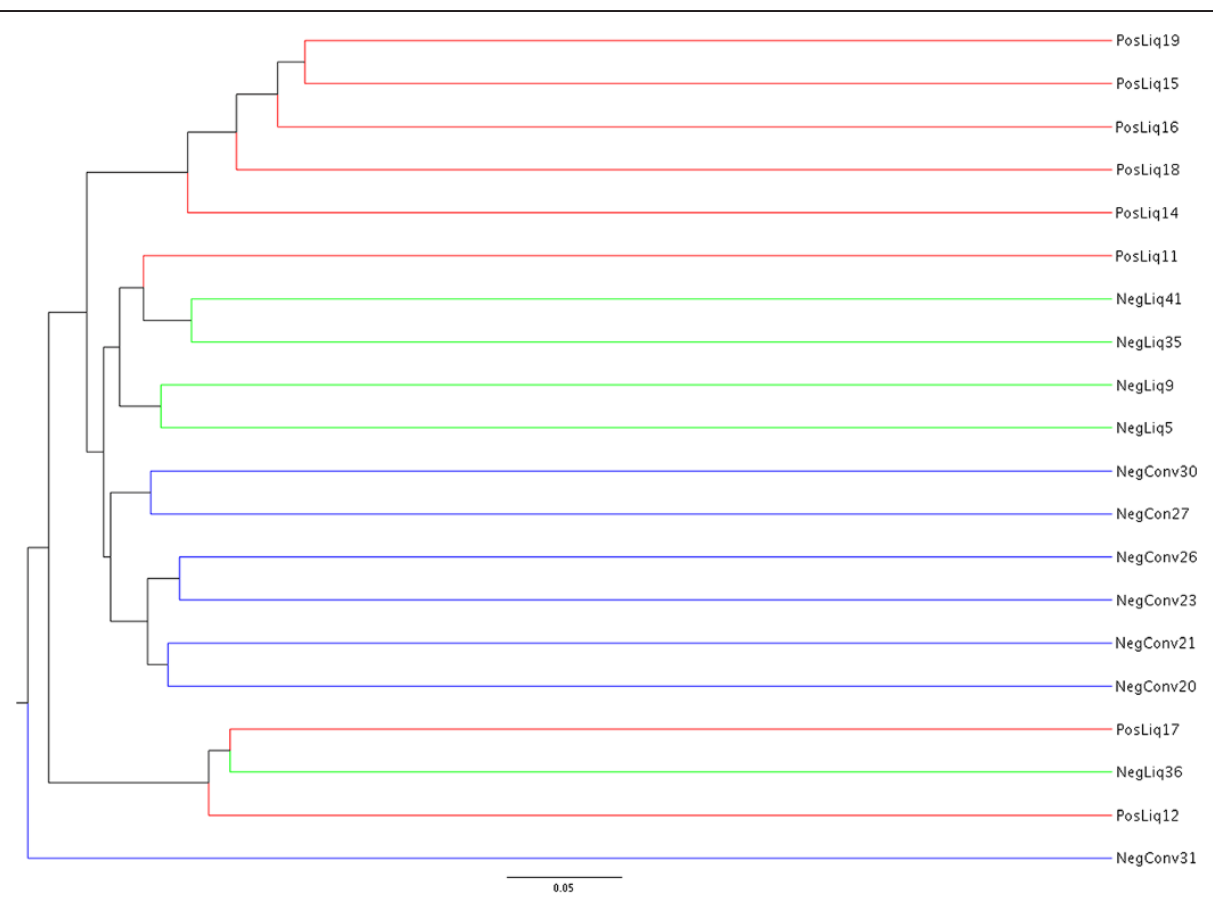

Figure 4 Dendrogram of the community membership of the nasal microbiota from twenty healthy pigs, based on the Jaccard index. (Legend: Conv = conventionally fed pigs, NegLiq = liquid fed pigs not carrying, PosLiq = liquid fed pigs carrying MRSA). 


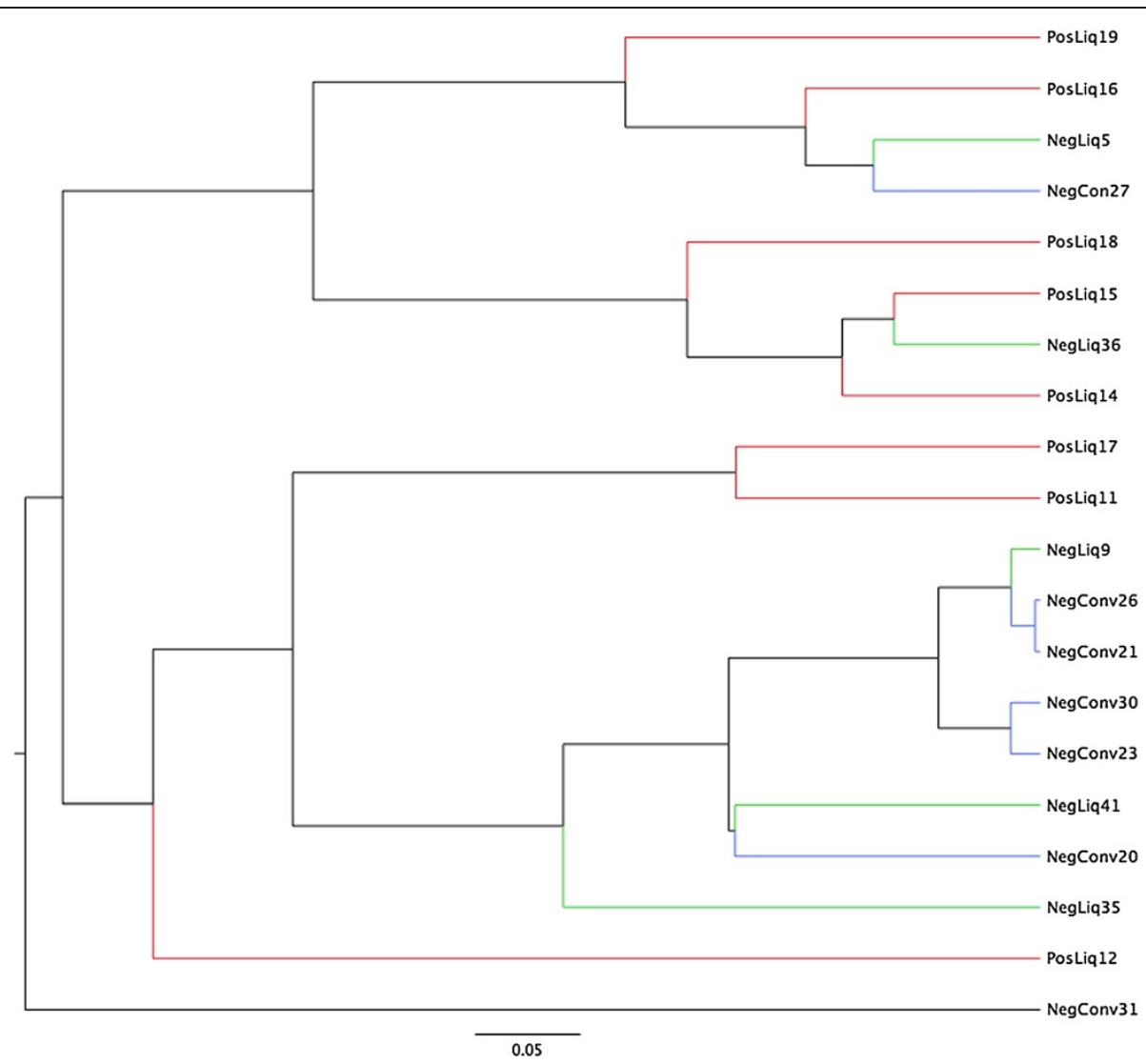

Figure 5 Dendrogram of the community structure of the nasal microbiota of twenty healthy pigs based on the Yue and Clayton index of dissimilarity. (Legend: Conv = conventionally fed pigs, NegLiq = liquid fed pigs not carrying MRSA, PosLiq = liquid fed pigs carrying MRSA).

Table 3 Indicator operational taxon units (OTUs) and their relative abundance (\%) for liquid-fed $(n=13)$ or conventionally-fed $(n=7)$ pigs

\begin{tabular}{ll}
\hline Group & Indicator (Phylum/Class/Order/Family/Genus) \\
\hline Liquid & Proteobacteria/Gammaproteobacteria/Pseudomonales/Moraxellaceae/Acinetobacter (3.8\%) \\
& Firmicutes/Bacilli/Lactobacillales/Leuconostoaceae/Leuconostoc (0.44\%) \\
& Firmicutes/Bacilli/Lactobacillales/Lactobacillaceae/Lactobacillus (2 OTUs) (0.33\%) \\
& Firmicutes/Bacilli/Lactobacillales/Aerococcaceae/Aerococcus (0.3\%) \\
& Proteobacteria/Gammaproteobacteria/Pseudomonadales/Pseudomonadaceae/Pseudomonas (11.7\%) \\
& Proteobacteria/Betaproteobacteria/Burkholderiales/Oxalobacteraceae/Janthinobacterium (3.8\%) \\
& Spirochaetes/Spirochaetes/Spirochaetales/Spirochaetaceae/Treponema (0.11\%) \\
& Proteobacteria/Gammaproteobacteria/Pseudomonales/Moraxellaceae/Moraxella (4 OTUs) (0.019\%) \\
& Firmicutes/Bacilli/Lactobacillales/Lactobacillaceae/Lactobacillus (0.077\%) \\
& Proteobacteria/Gammaproteobacteria/Pseudomonadales/Pseudomonadaceae/Pseudomonas (0.027\%) \\
& Proteobacteria/Unclassified (2 OTUs) (0.046\%) \\
& Firmicutes/Bacilli/Bacillales/Staphylococcaceae/Staphylococcus (0.28\%) \\
& Firmicutes/Bacilli/Bacillales/Staphylococcaceae/Unclassified (0.009\%) \\
& Firmicutes/Clostridia/Clostridiales/Ruminococcaceae/Unclassified (0.06\%) \\
& Firmicutes/Unclassified (0.0035\%) \\
\hline
\end{tabular}


tonsil, there were marked differences within Proteobacteria, as Actinobacillus and Pasteurella predominated in the study of porcine tonsillar tissue [2]. This could be the result of differences between the nasal passages and tonsil, as well as other factors such as pig age, farm management and laboratory methods.

Various differences were identified between pigs on the two farms. They differed in both their diet (liquid vs conventional) and antimicrobial exposure (tylosin vs no antimicrobials). Changes in the nasal microbiota could result from direct effects of the liquid feed (e.g. inoculation of microorganisms from feed into the nasal passages while eating) or indirect effects through modification of the fecal microbiota and altered environmental bacterial exposures. The impact of liquid whey feeding on the fecal microbiota was assessed in mature pigs [9]; but it was a cloning-based study that involved very small numbers of clones and provides little insight into the results of this study. Reasons for the significant lower relative abundance of Verrucomicrobia and Fibrobacteres is not clear. A decrease in Fibrobacteres is more understandable since this is a phylum of cellulose-degrading bacteria that would presumably be of less use in the intestinal tract of pigs fed liquid feeds. This difference may therefore indicate a change in the gut microbiota that is subsequently reflected in the nasal microbiota from environmental exposure. The other potential influence on the nasal microbiota is antimicrobial exposure of the liquid-fed group. Local or systemic effects could potentially modify the nasal microbiota, or could modify the fecal microbiota and indirectly impact the nasal microbiota.

Conflicting results were obtained for different tests of community structure and membership. A difference between feeding types was noted using the parsimony test and the Jaccard index. This can be visualized by assessment of the Jaccard tree, which also shows clustering of the MRSA-positive pigs. Yet, there was no significant difference in community structure based on analysis of the
Yue and Clayton tree, which includes relative abundance, and no difference with Unifrac or AMOVA. The relevance of conflicting results such as these is hard to interpret and could relate to differences in the statistical measures. Specifically, differences in rare OTUs with relative stability of abundant OTUs would have more of an impact of the Jaccard index (which only assesses community membership, not relative abundance). Limitations in statistical power must also be considered. While there was not agreement between all tests and there were many similarities between groups, some differences between pigs that were, or were not, carrying MRSA were noted, as were differences between pigs on different diets. These results indicate that further study of these differences to better characterize them and try to discern cause vs effect are indicated. The relative impact of diet and antimicrobial exposure cannot be discerned with this study design, and studies targeting specific management aspects are required.

There was a limited core microbiota, with no OTUs found in all samples and only one (accounting for $20 \%$ of sequences) found in $85 \%$ of samples. This is somewhat similar to humans, where only 2 OTUs, accounting for $17 \%$ of sequences, were identified in the anterior nares [8].

Indicator analysis is an ecological tool that is used to identify species that define an environment. By evaluating species fidelity (relative abundance of the species in the population) and exclusivity, species-habitat associations can be identified beyond simply comparing relative abundances. Numerous indicator OTUs were identified here, indicating bacterial groups that deserve further study to clarify their association with diet or MRSA status and reasons for that. Interestingly, OTUs from Firmicutes were the main indicators of MRSA negative pigs, including Lactobacillus and another Lactobacillaceae and, perhaps surprisingly, Staphylococcus. Whether these might be protective for MRSA colonization cannot be stated with these data but these results raise interesting questions since repopulation of the nasal microbiota with these species (e.g. 
through feed) could represent a practical intervention to reduce MRSA carriage. Further study of staphylococci, perhaps by using PCR targets with greater staphylococcal species resolution (e.g. $\operatorname{sod} A$ ) might provide more insight into this potential protective effect. These results also show that evaluation of indicator species may provide more insight than simply comparing relative abundances between groups.

Numerous genera were over- or under-represented between MRSA positive and negative, or conventionallyor liquid-fed pigs. These are too numerous to discuss, but they, along with indicator species, provide the basis for consideration and further study of individual components of the microbiota that might be influenced by diet and/or influence MRSA status. Many other genera had P values between 0.05 and 0.1 (data not presented), indicating that larger study might identify more organisms of interest. At the same time, though, the number of statistical comparison that were performed needs to be considered, as some statistically significant differences could be generated by random chance with such a large number of comparisons. Nonetheless, these data can form the starting point for further studies.

Within-farm matching was desired because of the unknown potential for inter-farm variation. A complicating factor in this study was the difficulty in identifying farms where both positive and negative pigs of the target age were present, since an 'all-or-nothing' effect was apparent on many farms. This, along with poor DNA yield from some nasal swabs, ultimately limited the number of samples available for analysis. The small sample size must be considered and study of larger populations and different farms would be useful. The presence of two apparent outliers, with large proportions of Firmicutes sequences, may have further weakened statistical power. Nonetheless, this study provides useful information about the nasal microbiota in pigs and raises some points for further study of both the impact of diet on the nasal microbiota and the role of the nasal microbiota in MRSA carriage.

Given the behaviour of pigs and their environment, it is likely that the nasal microbiota consists of resident nasal commensals and transient environmental contaminants. That likely explains the finding of enteric organisms like Salmonella, yet those were of very low abundances and the predominant components of the nasal microbiota were Proteobacteria that are uncommon in the intestinal tract (and therefore presumably the barn environment). Fecal contamination of the nasal passages might explain the two outliers that had a large number of Firmicutes, given the predominance of this phylum in porcine feces [10].

\section{Conclusion}

The nasal passages of slaughter-age pigs harbour a very rich and diverse microbial population that can apparently be influenced by diet and/or farm management practices. While the impact of the microbiota on MRSA carriage is unclear, these data suggest that further study of the role of the nasal microbiota on MRSA carriage and the potential that the nasal microbiota could be specifically manipulated by diet or antimicrobials warrant further study.

\section{Methods}

\section{Study population and sample collection}

Pigs from four commercial swine operations on southern Ontario, Canada were enrolled. Pigs were sampled 12 weeks prior to slaughter and clinically normal. Nasal swabs were collected using rayon-tipped culture swabs. Paired swabs were collected. One swab was tested for the presence of MRSA through selective enrichment culture, as has been previously described [4]. The other was placed in a sterile glass tube and stored at $-80^{\circ} \mathrm{C}$ until MRSA culture results were obtained. The goal was to obtain farmmatched groups of MRSA-positive and MRSA-negative pigs for microbiota analysis. The study was approved by the University of Guelph Animal Care Committee.

\section{DNA extraction and quality control}

DNA extraction was performed using a commercial kit (E.Z.N.A. Stool DNA Kit, Omega Bio-Tek Inc., Doraville, Georgia, USA) following the manufacturer's "stool DNA protocol for pathogen detection". DNA quantity and quality were accessed by spectrophotometry (NanoDrop, Roche, Mississauga, Canada).

\section{S rRNA gene amplification and sequencing}

PCR amplification of the V4 region of the 16S rRNA gene was designed based on Caporaso et al. 2010 [11] using the primers forward S-D-Bact-0564-a-S-15 (5'-AYTGGGYDT AAAGNG-3') and reverse S-D-Bact-0785-b-A-18 (5'-TA CNVGGGTATCTAATCC-3'). The forward and reverse primers were designed containing an overlapping region of the forward and reverse Illumina sequencing primers (TCGTCGGCAGCGTCAGATGTGTATAAGAGACAG and GTCTCGTGGGCTCGGAGATGTGTATAAGAGA CAG, respectively) in order to anneal them to primers containing the Illumina adaptors plus the $8 \mathrm{bp}$ identifier indices (forward: AATGATACGGCGACCACCGAGA TCTACAC-index-TCGTCGGCAGCGTC; reverse: CA AGCAGAAGACGGCATACGAGAT-index-GTCTCGT GGGCTCGG). A $50 \mathrm{ul}$ reaction was performed with $25 \mu \mathrm{L}$ of Kapa2G Fast HotStart ReadyMix 2X (KapaBiosystems); $1.3 \mu \mathrm{L}$ of BSA (Bio-Rad); $18.9 \mu \mathrm{L}$ of PCR-grade $\mathrm{H}_{2} \mathrm{O}, 2 \mu \mathrm{L}$ of DNA template, and $0.4 \mu \mathrm{L}$ of both the forward and the reverse $16 \mathrm{~S}$ primers $(10 \mathrm{pmol} / \mu \mathrm{L})$. The following PCR conditions were used; $3 \mathrm{~min}$ at $94^{\circ} \mathrm{C}$ for denaturing, and $30 \mathrm{cy}-$ cles of $45 \mathrm{sec}$ at $94^{\circ} \mathrm{C}$ for denaturing, $60 \mathrm{sec}$ at $50^{\circ} \mathrm{C}$ for annealing and $90 \mathrm{sec}$ at $72^{\circ} \mathrm{C}$ for elongation followed by a 
final period of $10 \mathrm{~min}$ at $72^{\circ} \mathrm{C}$ and kept at $4^{\circ} \mathrm{C}$ until purification.

PCR products were evaluated by electrophoresis in $2 \%$ agarose gel and purified with the Agencourt AMPure XP (Beckman Coulter Inc, Mississauga, Ontario, Canada) by mixing $20 \mu \mathrm{L}$ of amplicon with $72 \mu \mathrm{L}$ of AMPure on a 96 well plate. After $5 \mathrm{~min}$ at room temperature, beads were separated and washed twice with $80 \%$ ethanol and eluted in $30 \mu \mathrm{L}$ of water. After purification samples were quantified by spectrophotometry (Nanorop, Roche, Mississauga, Canada) and normalized to a final concentration of $2 \mathrm{nM}$. Sequencing of the library pool was performed at the University of Guelph's Advanced Analysis Centre using an Illumina MiSeq (San Diego, USA) and 2×250 chemistry.

\section{Microbiota assessment}

The mothur package of algorithms (v1.32) was used for analysis [12]. Paired end reads were aligned. Sequences $>244$ bp or $<237$ bp in length, and those containing any ambiguous base calls or long runs ( $>8 \mathrm{bp}$ ) of holopolymers were removed, as were sequences that did not align with the correct region. Chimeras were detected using uchime [13] through mothur v1.31 rather than v1.32 because of a bug that was present in the mothur v1.32 at the time of analysis. Sequences from chloroplasts, mitochondria, Archaea and Eukaryotes were also removed. CatchAll was used to assess species richness [14].

Sequences were binned into operational taxon units (OTUs) at a 3\% (0.03) dissimilarity level, and taxonomy was assigned using the Silva 16S rRNA reference database (www.arb-silva.de) [15]. Student's t-test or ANOVA with Tukey's post hoc test were used for comparison of continuous data, with a $P$ value of $<0.05$ considered significant.

Subsampling was then performed to normalize sequence number for subsequent analyses. Coverage was assessed using Good's coverage. Population diversity was described using the inverse Simpson's index. Dissimilarity between the three groups was assessed through creation of dendrograms using the Yue \& Clayton measure of dissimilarity (a measure of community structure, which considers shared OTUs and their relative abundances) and traditional Jaccard index (a measure of community membership, which considers the number of shared OTUs, not their abundance). Figures were generated by FigTree v1.4.0 (http://tree.bio.ed.ac.uk/). Parsimony and unweighted unifrac [16] tests were applied to evaluate the impact of feeding type and MRSA status on microbial population structure. Principal component analysis (PCA) was also performed. Analysis of molecular variance (AMOVA), a non-parametric evaluation of genetic diversity, was also applied to compare groups. Comparisons between MRSA positive and MRSA negative pigs were restricted to pigs from the same liquid-feeding farm to avoid potential bias. Significant $(P<0.05)$ indicator OTUs were identified. The core microbiota was assessed through identification of OTUs present in all samples at a minimum relative abundance of $1 \%$.

\section{Availability of supporting data}

The data set supporting the results of this article are available at the MG-RAST metagenomics analysis server (project 8129 , http://metagenomics.anl.gov).

\section{Abbreviations}

AMOVA: Analysis of molecular variance; ANOVA: Analysis of variance; MRSA: Methicillin-resistant Staphylococcus aureus; OTU: Operational taxon unit.

\section{Competing interests}

The authors declare that they have no competing interests.

\section{Authors' contributions}

JSW and RF designed the study. MS provided input with study design, coordinated sampling and performed MRSA culture. MJ performed molecular studies. JSW performed data analysis and drafted the manuscript. All authors read and approved the final manuscript.

\section{Acknowledgement}

This study was funded by the National Pork Board.

\section{Author details}

'Department of Pathobiology and Centre for Public Health and Zoonoses, Ontario Veterinary College, University of Guelph, Guelph, Ontario N1G2W1, Canada. ${ }^{2}$ Department of Population Medicine, Ontario Veterinary College, University of Guelph, Guelph, Ontario N1G2W1, Canada.

Received: 5 December 2013 Accepted: 12 March 2014 Published: 15 March 2014

\section{References}

1. Woods GT, Jensen AH, Gossling J, Rhoades HE, Nickelson WF: The effect of medicated feed on the nasal microflora and weight gain of pigs. Can J Comp Med 1972, 36(1):49-54.

2. Lowe BA, Marsh TL, Isaacs-Cosgrove N, Kirkwood RN, Kiupel M, Mulks MH: Defining the "core microbiome" of the microbial communities in the tonsils of healthy pigs. BMC Microbiol 2012, 12:20

3. Voss A, Loeffen F, Bakker J, Klaassen C, Wulf M: Methicillin-resistant Staphylococcus aureus in pig farming. Emerging Infect Dis 2005, 11 (12):1965-1966.

4. Khanna T, Friendship R, Dewey C, Weese J: Methicillin resistant Staphylococcus aureus colonization in pigs and pig farmers. Vet Microbiol 2008, 128:298-303.

5. Broens EM, Graat EAM, van der Wolf PJ, van de Giessen AW, de Jong MCM: Prevalence and risk factor analysis of livestock associated MRSA-positive pig herds in The Netherlands. Prev Vet Med 2011, 102:41-49.

6. Van Duijkeren E, Ikawaty R, Broekhuizen-Stins MJ, Jansen MD, Spalburg EC, De Neeling AJ, Allaart JG, Van Nes A, Wagenaar JA, Fluit AC: Transmission of methicillin-resistant Staphylococcus aureus strains between different kinds of pig farms. Vet Microbiol 2008, 126:383-389.

7. van Rijen MM, Bosch T, Heck ME, Kluytmans JA: Meticillin-resistant Staphylococcus aureus epidemiology and transmission in a Dutch hospital. J Hosp Infect 2009, 72(4):299-306.

8. Huse SM, Ye Y, Zhou Y, Fodor AA: A core human microbiome as viewed through 16S rRNA sequence clusters. PLOS ONE 2012, 7(6):e34242.

9. Kobayashi Y, Itoh A, Miyawaki K, Koike S, Iwabuchi O, limura Y, Kobashi Y, Kawashima T, Wakamatsu J, Hattori A, Marakami H, Morimatsu F, Nakaebisu T, Hishinuma T: Effect of liquid whey feeding on fecal microbiota of mature and growing pigs. Anim Sci J 2011, 82(4):607-615.

10. Schmidt B, Mulder IE, Musk CC, Aminov RI, Lewis M, Stokes CR, Bailey M, Prosser JI, Gill BP, Pluske JR, Kelly D: Establishment of normal gut microbiota is compromised under excessive hygiene conditions. PLOS ONE 2011, 6(12):e28284.

11. Caporaso JG, Kuczynski J, Stombaugh J, Bittinger K, Bushman FD, Costello EK, Fierer N, Peña AG, Goodrich JK, Gordon II, Huttley GA, Kelley ST, Knights D, 
Koenig JE, Ley RE, Lozupone CA, McDonald D, Muegge BD, Pirrung M, Reeder J, Sevinsky JR, Turnbaugh PJ, Walters WA, Widmann J, Yatsunenko T, Zaneveld J, Knight R: QIIME allows analysis of high-throughput community sequencing data. Nat Methods 2010, 7(5):335-336.

12. Schloss PD, Westcott SL, Ryabin T, Hall JR, Hartmann M, Hollister EB, Lesniewski RA, Oakley BB, Parks DH, Robinson CJ, Sahl JW, Stres B, Thallinger GG, Van Horn DJ, Weber CF: Introducing mothur: open-source, platformindependent, community-supported software for describing and comparing microbial communities. Appl Environ Microbiol 2009, 75(23):7537-7541.

13. Edgar RC, Haas BJ, Clemente JC, Quince C, Knight R: UCHIME improves sensitivity and speed of chimera detection. Bioinformatics 2011, 27(16):2194-2200.

14. Bunge J, Woodard L, Bohning D, Foster JA, Connolly S, Allen HK: Estimating population diversity with CatchAll. Bioinformatics 2012, 28:1045-1047.

15. Quast C, Pruesse E, Yilmaz P, Gerken J, Schweer T, Yarza P, Peplies J, Glöckner FO The SILVA ribosomal RNA gene database project: improved data processing and web-based tools. Nucleic Acids Res 2013, 4:D590-D596.

16. Lozupone C, Hamady M, Knight R: UniFrac-an online tool for comparing microbial community diversity in a phylogenetic context. BMC Bioinforma 2006, 7:371.

doi:10.1186/1746-6148-10-69

Cite this article as: Weese et al:: Evaluation of the nasal microbiota in

slaughter-age pigs and the impact on nasal methicillin-resistant Staphylococcus aureus (MRSA) carriage. BMC Veterinary Research 2014 10:69.

\section{Submit your next manuscript to BioMed Central and take full advantage of:}

- Convenient online submission

- Thorough peer review

- No space constraints or color figure charges

- Immediate publication on acceptance

- Inclusion in PubMed, CAS, Scopus and Google Scholar

- Research which is freely available for redistribution 